\title{
Evaluation of interest rate-linked DLSs
}

\author{
Manduk Kim ${ }^{a}$, Seongjoo Song ${ }^{1, a}$ \\ ${ }^{a}$ Department of Statistics, Korea University, Korea
}

\begin{abstract}
Derivative-linked securities (DLS) is a type of derivatives that offer an agreed return when the underlying asset price moves within a specified range by the maturity date. The underlying assets of DLS are diverse such as interest rates, exchange rates, crude oil, or gold. A German 10-year bond rate-linked DLS and a USD-GBP CMS rate-linked DLS have recently become a social issue in Korea due to a huge loss to investors. In this regard, this paper accounts for the payoff structure of these products and evaluates their prices and fair coupon rates as well as risk measures such as Value-at-Risk (VaR) and Tail-Value-at-Risk (TVaR). We would like to examine how risky these products were and whether or not their coupon rates were appropriate.

We use Hull-White Model as the stochastic model for the underlying assets and Monte Carlo (MC) methods to obtain numerical results.

The no-arbitrage prices of the German 10-year bond rate-linked DLS and the USD-GBP CMS rate-linked DLS at the center of the social issue turned out to be $0.9662 \%$ and $0.9355 \%$ of the original investment, respectively. Considering that Korea government bond rate for 2018 is about $2 \%$, these values are quite low. The fair coupon rates that make the prices of DLS equal to the original investment are computed as $4.76 \%$ for the German 10-year bond rate-linked DLS and 7\% for the USD-GBP CMS rate-linked DLS. Their actual coupon rates were $1.4 \%$ and $3.5 \%$. The $95 \% \mathrm{VaR}$ and TVaR of the loss for German 10-year bond rate-linked DLS are $37.30 \%$ and $64.45 \%$, and those of the loss for USD-GBP CMS rate-linked DLS are $73.98 \%$ and $87.43 \%$ of the initial investment. Summing up the numerical results obtained, we could see that the DLS products of our interest were indeed quite unfavorable to individual investors.
\end{abstract}

Keywords: interest rate-linked derivative-linked securities, pricing, coupon rate, Hull-White model, Value-at-Risk, Tail-Value-at-Risk

\section{Introduction}

Derivative-linked securities (DLS) is a financial instrument that offers an agreed return when the underlying asset price moves within a specified range by a fixed maturity date. It is similar to Equitylinked securities (ELS), but the types of underlying assets are different. The return of DLS is linked to interest rates, exchange rates, crude oil, gold, and so on, while the return of ELS is linked to stocks or market indices. In year 2019, the German 10-year bond rate-linked DLS that matured on Sept. $26^{\text {th }}, 2019$ and the USD-GBP CMS rate-linked DLS that matured on Sept. $25^{\text {th }}, 2019$ have inflicted enormous losses to investors and thus, these products have become a big social issue in Korea. It was also suspected that their risks had not been properly informed to some investors when they were

\footnotetext{
This work was supported by the National Research Foundation of Korea(NRF) grant funded by the Korea government(MIST) (No.2021R1F1A1048986).

This manuscript is based on the first author, Manduk Kim's Master thesis from Korea University.

${ }^{1}$ Corresponding author: Department of Statistics, Korea University, 145 Anam-ro, Seongbuk-gu, Seoul 02841, Korea.

E-mail: sjsong@korea.ac.kr
}

Published 31 January 2022 / journal homepage: http://csam.or.kr

(C) 2022 The Korean Statistical Society, and Korean International Statistical Society. All rights reserved. 
sold. In this paper, we would like to analyze how fair these two products were designed and how risky they were in the market situation of that time. Below, the German 10-year bond rate-linked DLS we consider will be referred as G10Y DLS and the USD-GBP CMS rate-linked DLS of interest as UGCMS DLS.

The total amount of principals of G10Y DLS and UGCMS DLS was 822.4 billion KRW, which is approximately 680 million USD at the exchange rate of that time. The guaranteed coupon rates for G10Y DLS and UGCMS DLS were $1.4 \%$ and 3.5\%, respectively. These DLSs were classified as highly risky instruments with a high probability of losing principal at the time of sales. Nevertheless, it was quite surprising that the realized rate of return of G10Y DLS with maturity, September 26, 2019 was $-98.6 \%$ and that of UGCMS DLS with maturity, September 25, 2019 was $-46.46 \%$.

Accurate pricing and determination of proper coupon rates of DLSs would be necessary for the formulation of a sound and healthy financial market. In this sense, studies of DLSs should be done in both the investors' view and the financial instrument sellers' view. From the perspective of investors, if the price of DLS was correctly assessed, appropriate investments in DLS that reflect each investor's risk-taking property would be possible. From the perspective of financial instrument sellers, the fair coupon rate of DLS would lead to the development of stable instruments providing a proper level of return. The general structure of DLSs is quite asymmetric in the sense that the loss on the side of sellers is limited by the guaranteed coupon rate but the loss on the investors could be the whole investments.

While there have been many studies on ELS in finance literature, not much research on DLS has been done. For examples of studies on ELS, Broadie et al. (1997) suggested a continuity correction for discrete barrier options, which can be applied to path dependent options like ELS options. Lyuu (1998) extended combinatorial methods to pricing European-style barrier options, which could be used for numerical pricing of ELS products. Jeong et al. (2010) presented the numerical valuation of the two-asset step-down equity-linked securities option by the operator-splitting method. Lee et al. (2019) explored the pricing of ELS products through the icicled step-barrier option pricing formula. In this paper, we focus on DLSs that have recently emerged as significant products in financial markets and evaluate the prices and coupon rates that make the prices equal to the original investment by Monte Carlo simulations. Moreover, we estimate risk measures such as Value-at-Risk (VaR) and TailValue-at-Risk (TVaR) of losses by Monte Carlo simulations so that we can figure out the proper levels of risk.

G10Y DLS and UGCMS DLS are both interest rate-linked DLSs, and thus, we need to specify a stochastic model for the underlying interest rate. We assume that the interest rate follows Hull-White model, which is one of the most widely used models for pricing interest rate derivatives. For instances, Gupta and Subrahmanyam (2005) examined the pricing and hedging performance of interest rate models including Hull-White model using US dollar cap and floor daily prices. Henrard (2009) proposed an approximation for the pricing of European swaption under Hull-White model. Ostrovski (2013) described how an efficient and exact Monte Carlo simulation of Hull-White model could be performed. One thing to note is that we need to use the risk-neutral measure for pricing and finding the fair coupon rates but use the real-world probability measure for computing risk measures. Hull-White model is defined under the risk-neutral measure, so we need to modify parameters to incorporate the market price of risk for computing risk measures.

The remainder of the paper is organized as follows. Section 2 first explores the current trend of DLSs traded in financial markets in South Korea. Then we describe the payoff structures of the one-asset DLS, G10Y DLS and the two-asset DLS, UGCMS DLS. Section 3 focuses on Hull-White model as the stochastic model for the underlying interest rates and introduces risk measures, VaR and 
Table 1: Issuance status of ELS \& DLS in Korean financial market from 2014 to 2019(Unit of volume: Billion KRW)

\begin{tabular}{|c|c|c|c|c|}
\hline \multirow[b]{3}{*}{ Year } & \multicolumn{4}{|c|}{ Issue } \\
\hline & \multicolumn{2}{|c|}{ ELS } & \multicolumn{2}{|c|}{ DLS } \\
\hline & \# of Contracts & Volume & \# of Contracts & Volume \\
\hline 2014 & 17,089 & 51,617 & 2,072 & 10,639 \\
\hline 2015 & 18,865 & 61,286 & 2,168 & 11,859 \\
\hline 2016 & 16,068 & 34,624 & 2,863 & 16,074 \\
\hline 2017 & 21,283 & 65,085 & 4,186 & 18,400 \\
\hline 2018 & 21,230 & 65,941 & 3,368 & 16,306 \\
\hline 2019 & 22,178 & 76,732 & 3,545 & 17,603 \\
\hline
\end{tabular}

Table 2: Volume of DLS by underlying assets in Korean financial market in the third quarter, 2019 (Unit : Trillion KRW): Values in parentheses are percentages

\begin{tabular}{ccccccc}
\hline \hline Underlying asset & Interest rate & Credit & Exchange rate & Commodity goods & Etc & Total \\
Volume & $2.0(30.7)$ & $1.3(20.0)$ & $0.4(6.0)$ & $0.1(1.5)$ & $2.6(40.0)$ & $6.5(100.0)$ \\
\hline \hline
\end{tabular}

TVaR. Section 4 concerns with the pricing and the computing risk measures of DLSs using Monte Carlo simulations. Section 5 concludes the paper with some future considerations.

\section{Derivative-linked securities (DLS)}

Recently, low interest rates have continued globally, so investors who want higher coupon rates turn to other investment possibilities such as ELS or DLS instead of traditional fixed income securities. Table 1 shows the number of contracts and the volume of ELS and DLS products sold in South Korea from 2014 to 2019. The number of contracts and the volume of DLS in 2014 was $12 \%$ and $20 \%$ of those of ELS, respectively. These figures has risen to $16 \%$ and $23 \%$ in 2019. Although DLS still accounts for a smaller portion of the financial market than ELS, DLS has been on the rise in the number of cases and the volume. Table 2 provides the volume of DLS for each underlying asset issued in the third quarter of 2019. The numbers in parentheses are the percentage of DLS issued for the corresponding underlying asset. The highest volume is $30.7 \%$, which corresponds to the interest rate-linked DLS. We can see that interest rate is currently the main underlying asset in DLS. Sections 2.1 and 2.2 will discuss interest rate-linked DLS in more details by introducing the payoff structures of G10Y DLS and UGCMS DLS. Note that other DLS products can also be analysed similarly, although they may have slightly different payoff structures.

\subsection{One-asset Interest rate-linked DLS : German 10-year bond rate}

One of the interest rate-linked DLS that became a social issue in Korea in 2019 is the German 10-year bond rate-linked DLS (G10Y DLS), which has the payoff structure in (2.1). Here, we assume that the initial investment is one unit; that is, when we invest one unit on the starting date, at the maturity, we get (2.1) returned. G10Y DLS was issued with the starting date of May 26, 2019 and the maturity date of September 26, 2019. The maturity date refers to the termination of the contract of DLS. The 


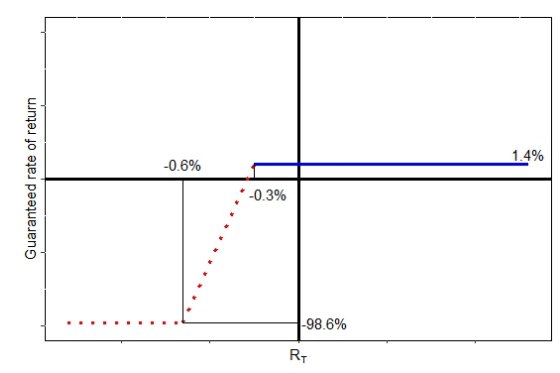

Figure 1: Plot of payoff structure of the German $10 Y$ bond rate-linked DLS.

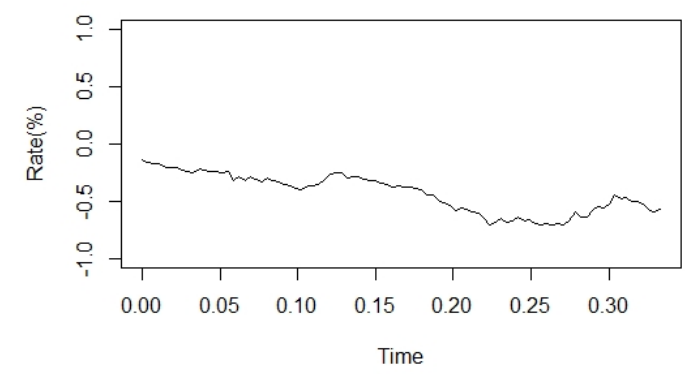

Figure 2: German 10Y bond rate from May 27, 2019 to September 25, 2019.

final valuation of the underlying asset was done on September 25, 2019.

$$
\text { Payoff }= \begin{cases}1+0.014, & R_{T} \geq-0.003 \\ {\left[2+\frac{1000}{3} R_{T}\right]+0.014,} & -0.006 \leq R_{T}<-0.003 \\ 0.014, & R_{T}<-0.006\end{cases}
$$

where $R_{T}$ denotes the German 10-year bond rate per annum at the closing time of the Korean market on the valuation date, September 25, 2019 and $T$ is 1/3 (years), which is the term of this product. When the principal of $P v$ is invested at the starting date, then $P v \times(2.1)$ is returned on the maturity date. $0.014(1.4 \%)$ is the coupon rate proposed by the financial institution that sold G10Y DLS. Figure 1 illustrates this payoff structure graphically by visualizing the rate of return and values of $R_{T}$. The horizontal axis represents $R_{T}(\%)$ and the vertical axis represents the corresponding rate of return (\%). If $R_{T}$ is greater than $-0.3 \%$, the constant rate of $1.4 \%$ of the original investment is paid as the return of the investment. If $R_{T}$ ends up with less than $-0.3 \%$, the rate of return starts to decrease and at $-0.6 \%$, the minimum value of $-98.6 \%$ is reached. For example, if $R_{T}=-0.2 \%, 1.4 \%$ of the investment is returned as the profit and if $R_{T}=-0.5 \%, 1000 / 3 \times(-0.005)+1.014=-65.3 \%$ of the investment is returned as the profit. In other words, $34.7 \%$ of the principal investment will be lost. If $R_{T}<-0.006,98.6 \%$ of the principal will be lost. The coupon rate $1.4 \%$ was somewhat attractive compared to the prevailing interest rate in year 2019. However, from Figure 1, we can easily see that the payoff structure possibly brings a huge loss depending on the value of $R_{T}$.

Figure 2 displays the German 10-year bond rate observed from May 27, 2019, to September 25, 


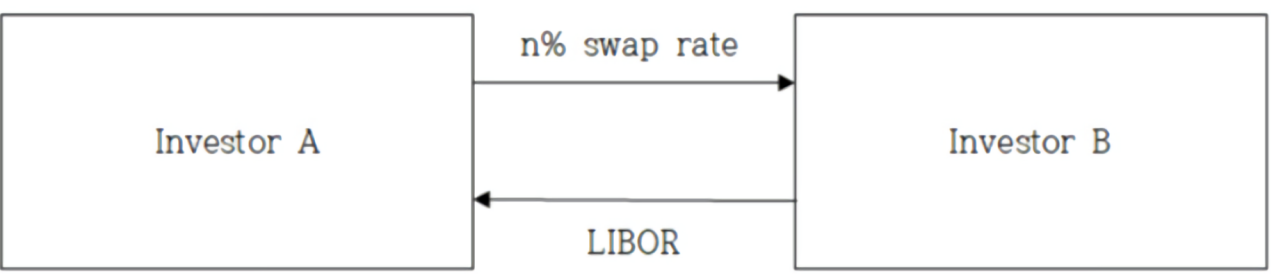

Figure 3: Interest rate swap between two investors.

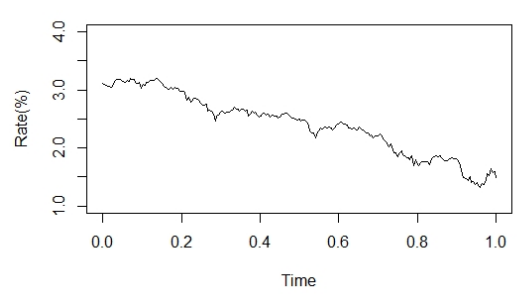

(a) USD 5 Y CMS rate

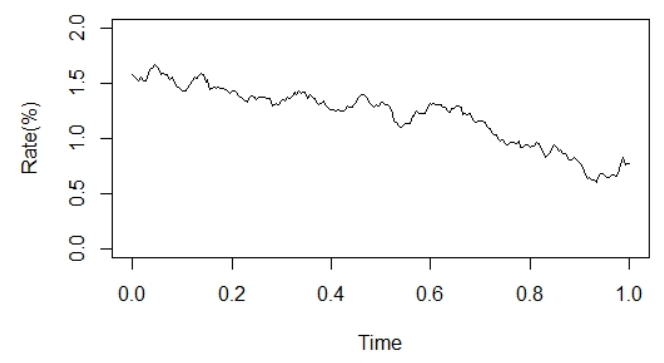

(b) GBP 7 Y CMS rate

Figure 4: CMS rates from September 25, 2018 to September 20, 2019.

2019. We can see that the rate starts at $-0.142 \%$, decreases over time and finally reaches $-0.576 \%$. The $x$-axis is the time measured in years. Note that these are the rates observed daily at the closing time of the German market, so $-0.576 \%$ is not $R_{T}$ in equation (2.1) to calculate the actual loss of G10Y DLS. The German 10-year bond rate applied to G10Y DLS, which is $R_{T}$ in equation (2.1) was $-0.619 \%$, which was the rate observed at the closing time of the Korean market on the final valuation date (the actual percentage of loss includes the settlement of the asset management fee, which is ignored in this study).

\subsection{Two-asset interest rate-linked derivative-linked securities : USD-GBP CMS rate}

First of all, let us introduce a constant maturity swap (CMS). A swap is an agreement between two investors to exchange a cash flow in the future. The most common type of swap is the interest rate swap, which is an agreement between two investors that exchanges a fixed rate and a floating rate. In Figure 3, investors $\mathrm{A}$ and $\mathrm{B}$ exchange $n \%$ fixed rate and LIBOR (London Interbank Offered Rate) where LIBOR is the most commonly used floating rate and $n \%$ is called the swap rate. That is, investor $\mathrm{B}$ converts the cash flow on interest payments from a floating rate to a fixed rate through this swap.

CMS is a variation of the interest rate swap in which the maturity of floating portion is periodically reset. At each time of the interest payments, the investors exchange interests based on the $c$-year swap rate and LIBOR or a fixed rate, where $c$ is the maturity of the swap rate used. In general, the swap rates have a longer maturity than the reset period. The $c$-year swap rate to be used for the floating portion has the same maturity at all times of interest payments and is called CMS rate. For example, USD 5-year swap rate can be used for CMS with the reset period of 3 months. LIBOR is commonly used for the other portion of CMS, but a fixed rate or another CMS rate can also be used. The CMS 


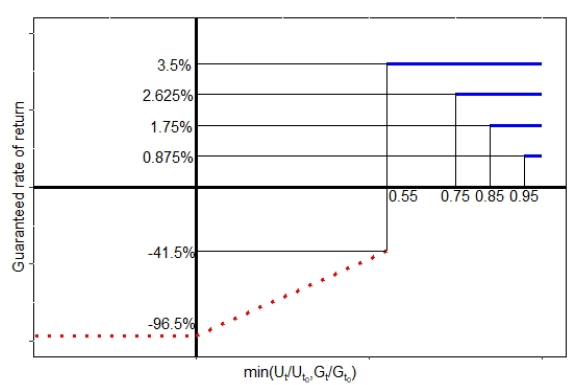

Figure 5: Payoff structure of the USD-GBP CMS rate-linked DLS issued on September 25, 2018.

with USD 5-year swap rate for the floating portion and is called USD 5-year CMS.

The two-asset DLS we consider in this paper is the USD-GBP CMS rate-linked DLS with the underlying assets of USD 5-year CMS and GBP 7-year CMS. As mentioned in Section 1, we abbreviate the name of this two-asset interest rate-linked DLS as UGCMS DLS. Figure 4(a) and 4(b) are plots of the USD 5-year CMS rate and GBP 7-year CMS rate from September 25, 2018, to September 25, 2019, respectively. Since the maturity of UGCMS DLS is 1 year, CMS rates for 1 year are displayed. USD 5-year CMS rate starts at 3.112\%, decreases over time and finally reaches $1.488 \%$, while GBP 7-year CMS rate starts at $1.578 \%$, decreases over time and finally reaches $0.705 \%$. These rates also have decreasing trend as German 5-year bond rate, but they stay positive. As with German 10Y bond rates, these are the rates observed daily at the closing time of markets overseas. So they are not the input values in the payoff function in (2.2). In the payoff function, the observed rates at the closing time of Korean market on the valuation date is to be used. Unfortunately, we could only find the daily closing CMS rates overseas.

UGCMS DLS was issued with the starting date of September 25, 2018 and the maturity date of September 25, 2019. The valuation date was September 24, 2019. The payoff function of UGCMS DLS is given in (2.2). Again, we assume that the initial investment is one unit.

$$
\text { Payoff }= \begin{cases}1+0.035 t_{1}\left(\text { Early redemption at } t_{1}\right), & \text { if the realized path } \in A \\ 1+0.035 t_{2}\left(\text { Early redemption at } t_{2}\right), & \text { if the realized path } \in A^{c} \cap B \\ 1+0.035 t_{3}\left(\text { Early redemption at } t_{3}\right), & \text { if the realized path } \in A^{c} \cap B^{c} \cap C \\ 1+0.035, & \text { if the realized path } \in A^{c} \cap B^{c} \cap C^{c} \cap D \\ \min \left(\frac{U_{t_{4}}}{U_{t_{0}}}, \frac{G_{t_{4}}}{G_{t_{0}}}\right)+0.035, & \text { if the realized path } \in A^{c} \cap B^{c} \cap C^{c} \cap D^{c} \cap E \\ 0.035, & \text { otherwise }\end{cases}
$$

where $U_{t}$ and $G_{t}$ denote the USD CMS rate and the GBP CMS rate per annum at time $t, 0<t \leq 1$. $t_{0}=0$ indicates the starting date of the contract, September 25, 2018 and $t_{4}$ is the valuation date, September 24, 2019. $t_{i}$ 's $i=1,2,3$ are the dates when the underlying rates are evaluated: $t_{1}=1 / 4$, $t_{2}=1 / 2, t_{3}=3 / 4$ years from the starting date. There will be early redemptions at $t_{1}, t_{2}$, or $t_{3}$ if the realized path is in $A \cup B \cup C$, and otherwise, the payment is made at the maturity. The sets are defined as $A=\left\{0.95 \leq \min \left(U_{t_{1}} / U_{t_{0}}, G_{t_{1}} / G_{t_{0}}\right)\right\}, B=\left\{0.85 \leq \min \left(U_{t_{2}} / U_{t_{0}}, G_{t_{2}} / G_{t_{0}}\right)\right\}, C=\{0.75 \leq$ $\left.\min \left(U_{t_{3}} / U_{t_{0}}, G_{t_{3}} / G_{t_{0}}\right)\right\}, D=\left\{0.55 \leq \min \left(U_{t_{4}} / U_{t_{0}}, G_{t_{4}} / G_{t_{0}}\right)\right\}, E=\left\{0 \leq \min \left(U_{t_{4}} / U_{t_{0}}, G_{t_{4}} / G_{t_{0}}\right)<0.55\right\}$. Note that as long as the realized path is included in $A \cup B \cup C \cup D$, the annualized rate of return is the same as $3.5 \%$, which is the coupon rate of this DLS product. As with G10Y DLS, this product also 
has a possibility of a big loss, with the maximum loss of $96.5 \%$ of the entire investment. One thing to note is that $U_{t_{i}}$ and $G_{t_{i}}, i=0, \ldots, 4$ are the daily CMS rates at the closing time of the Korean market.

Figure 5 illustrates the payoff structure of UGCMS DLS graphically. The horizontal axis represents $\min \left(U_{t} / U_{t_{0}}, G_{t} / G_{t_{0}}\right)$ and the vertical axis represents the coresponding rate of return (\%). If $\min \left(U_{t} / U_{t_{0}}, G_{t} / G_{t_{0}}\right)$ is greater than or equal to 0.95 at $t_{1}, 0.875 \%$ of the principal is returned at $t=t_{1}$ along with the original principal. Otherwise, the opportunity for early redemption is given again at $t_{2}$. If $\min \left(U_{t} / U_{t_{0}}, G_{t} / G_{t_{0}}\right)$ is greater than or equal to 0.85 at $t_{2}, 1.75 \%$ of the principal is returned at $t=t_{2}$ along with the original principal, and so on. When early redemptions are not made and if $\min \left(U_{t} / U_{t_{0}}, G_{t} / G_{t_{0}}\right)$ is between 0 and 0.55 at $t_{4},\left(100 \min \left(U_{t_{4}} / U_{t_{0}}, G_{t_{4}} / G_{t_{0}}\right)-96.5\right) \%$ of the principal is returned at the maturity along with the original principal. The rate of return will not go below $-96.5 \%$. For example, if early redemptions are not made and $\min \left(U_{t_{4}} / U_{t_{0}}, G_{t_{4}} / G_{t_{0}}\right)$ is 0.3 , then $0.3-0.965=-0.665$ and thus, $66.5 \%$ of the original principal will be lost.

\section{Interest rate models and risk measures}

Since the DLSs we consider are both interest rate-linked DLSs, we need a stochastic model for explaining the underlying interest rates. There are two types of interest rate models, the equilibrium model and the no-arbitrage model. Equilibrium model derives a stochastic process for the short rate that applies to an infinitesimally short period of time based on some assumptions about economic variables. Short rate is also referred as the instantaneous spot rate. Commonly used equilibrium models are Rendleman and Barter model, Vasicek model, and Cox-Ingersoll-Ross model. Rendleman and Barter (1980) developed a model in which interest rates follow a stochastic process similar to stock prices processes. However, stock prices do not have the phenomenon of mean reversion that is a wellknown characteristic of interest rate processes. The interest rates tend to pull back to some long-run average level over time, which is called as the mean reversion. Thus, Rendelman and Barter model does not reflect the mean reversion of interest rate processes. Vasicek (1977) developed a model that incorporates the mean reversion and Cox et al. (1985) extended Vasicek model to obtain CIR model with the diffusion parameter proportional to the square root of the current short rate. In CIR model, when interest rate increases in the short term, the standard deviation of interest rate also increases, while the standard deviation of interest rate is always constant in Vasicek model. Note that the interest rate can become negative with Vasicek model but cannot become negative with CIR model.

No-arbitrage models are models desinged to be exactly consistent with the current term structure of interest rates. Suppose $R(t, T)$ refers the spot interest rate at time $t$ for maturity $T$, which means that $R(t, T)$ can be used to price bonds at $t$ that matures in $T-t$ years. The term structure of interest rate at time $t$ is roughly the relationship between $R(t, T)$ and its maturity, $T$. That is, it is the curve that corresponds $R(t, T)$ to $T$ for $T>t$. The disadvantage of equilibrium models is that they do not fit the current term structure since the term structure is the output of the model. When the model does not price bonds correctly due to the incorrect term structure, even if the error is small, bond option prices could be quite largely wrong. With no-arbitrage models, on the other hand, the term structure is an input, so the observed term structure from the market is consistent with the term structure derived from the model.

Ho-Lee model (Ho and Lee, 1986) is the first no-arbitrage model introduced but more widely used no-arbitrige model is Hull-White model. Ho-Lee model is easy to apply, but there is not much flexibility in choosing the volatility structure and it does not incorporate the mean-reversion. Hull and White (1990) develped a model by generalizing the level of mean reversion from Vasicek model. While Vasicek model has a constant level of mean reversion, Hull-White model has a time-varying 
level of mean reversion so that it provides an exact fit to the initial term structure. We explore more about Hull-White model in Section 3.1 and will use Hull-White model in Section 4 as the underlying process of interest rates.

\subsection{Hull-White model}

We suppose that the underlying asset processes are defined on a probability space, $\left(\Omega, \mathcal{F},\left(\mathcal{F}_{t}\right)_{t \geq 0}, Q\right)$. $\left(\mathcal{F}_{t}\right)$ is a filtration generated by the underlying asset processes, containing accumulated information up to time $t$. Assume that the interest rate follows Hull-White model given below under the risk-neutral measure $Q$,

$$
d r_{t}=\left[\theta(t)-\alpha r_{t}\right] d t+\sigma d z_{t}
$$

where $r_{t}$ refers to the short rate at time $t$ and $\alpha$ and $\sigma$ are constants. $z$ is the standard Brownian motion under the risk-neutral measure. $\alpha$ is the rate of mean reversion, which indicates that $r_{t}$ tends to converge to its average over time. Larger $\alpha$ means a faster mean reversion. In the model, $r_{t}$ returns to the $\theta(t) / \alpha$ at the speed of $\alpha . \sigma$ is the volatility of interest rate and $\theta(t)$ is a time-varying quantity that is calculated using the initial term structure.

Suppose that $F(0, t)$ is the instantaneous forward rate for a maturity $t$ at time zero. Using equation (3.1), we derive the relationship between $\theta(t)$ and $F(0, t)$ as

$$
\theta(t)=\frac{\partial F(0, t)}{\partial t}+\alpha F(0, t)+\frac{\sigma^{2}}{2 \alpha}\left(1-e^{-2 \alpha t}\right)
$$

$F(0, t)$ is calculated from $P(0, t)$ by using the equation (3.3). $P(0, t)$ is a bond price at time 0 with maturity $t$.

$$
F(0, t)=-\frac{\partial \ln P(0, t)}{\partial t}
$$

And $P(0, t)$ is calculated from $R(0, t)$, the spot interest rate prevailing at time 0 for maturity $t$, using the following equation (3.4),

$$
P(0, t)=e^{-R(0, t) t}
$$

Therefore, the initial term structure, $R(0, t)$ is used to calculate $\theta(t)$ through equations (3.1), (3.3), and (3.4). Under Hull-White model, $r_{t} \mid \mathcal{F}_{s}$ follows a normal distribution with the mean $\mu$ and the variance $\gamma^{2}$ given below,

$$
r_{t} \mid \mathcal{F}_{s} \sim N\left(\mu, \gamma^{2}\right)
$$

where

$$
\begin{aligned}
\mu & =r_{s} e^{-\alpha(t-s)}+F(0, t)+\frac{\sigma^{2}}{2 \alpha}\left(1-e^{-\alpha t}\right)^{2}-F(0, s) e^{-\alpha(t-s)}-\frac{\sigma^{2}}{2 \alpha}\left(1-e^{-\alpha s}\right)^{2} e^{-\alpha(t-s)}, \\
\gamma^{2} & =\frac{\sigma^{2}}{2 \alpha}\left(1-e^{-2 \alpha(t-s)}\right) .
\end{aligned}
$$

This fact on the distribution of the interest rate is useful when we generate interest rate paths in Monte-Carlo simulations. Bond price with maturity date $T$ is determined by the following,

$$
P(t, T)=A(t, T) e^{-B(t, T) r_{t}},
$$




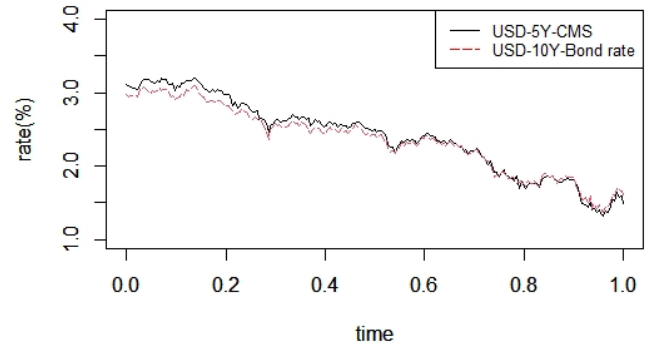

(a) USD $5 Y$ CMS rate, USD $10 Y$ bond rate

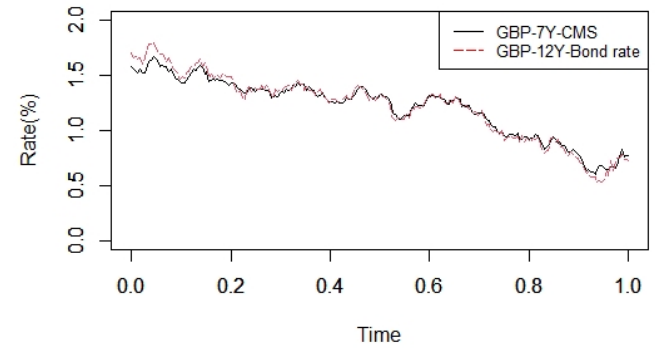

(b) GBP $7 Y$ CMS rate, GBP $12 Y$ bond rate

Figure 6: CMS rates and bond rates of USD and GBP from September 25, 2018 to September 24, 2019.

where

$$
\begin{aligned}
B(t, T) & =\frac{1-e^{-\alpha(T-t)}}{\alpha}, \\
\ln A(t, T) & =\ln \frac{P(0, T)}{P(0, t)}+B(t, T) F(0, t)-\frac{1}{4 \alpha^{3}} \sigma^{2}\left(e^{-\alpha T}-e^{-\alpha t}\right)^{2}\left(e^{2 \alpha t}-1\right) .
\end{aligned}
$$

For more details on Hull-White model, one can refer, for example, Hull (2011).

In the case of UGCMS DLS, we use the bond price formula, (3.8) for CMS rates calculated from the model. Figure 6(a) and 6(b) compare USD 5Y CMS rates and GBP 7Y CMS rates with USD 10Y bond rates and GBP 12Y bond rates, respectively from September 25, 2018, to September 24, 2019. We can see that it would be reasonable to apply bond price formula to calculate CMS rates from the model since CMS rates and bond rates are very similar.

\subsection{Risk measures}

Value at Risk (VaR) is a statistical measure of the riskiness of financial entities or portfolios of assets. It is defined as the maximum dollar amount expected to be lost, at a predefined confidence level. For example, $99 \%$ VaR of 1 million KRW means that there is $99 \%$ confidence that the portfolio will not lose more than 1 million KRW. It can be written in the following equation.

$$
P\left(X>\pi_{p}\right)=1-p
$$

where $X$ is a loss random variable and $\pi_{p}$ is the $100 p \%$ VaR of $X$. Although Value at Risk is an easy and intuitive risk measure, it is considered insufficient to reflect the current level of risk. Value at Risk does not tell us anything about how big the loss will be when the loss is larger than the VaR computed. Also, it does not satisfy the subadditivity condition of a coherent risk measure. It means that Value at Risk does not fully explain the diversification effect that is widely accepted in finance literature and industry.

There are several coherent risk measures proposed in finance literature, and the most widely used one is Tail-Value at Risk (TVaR). 100p\% TVaR is the expected loss on the condition that loss exceeds the $100 p \%$ VaR of $X$, given as in equation (3.12). For example, 99\% TVaR of 1 million KRW means that the expected loss is 1 million KRW given that the loss is greater than $\pi_{0.99}$. Refer to Kulgman $e t$ 
al. (2012) for more information on VaR and TVaR.

$$
\operatorname{TVaR}_{p}(X)=E\left(X \mid X>\pi_{p}\right) .
$$

We will try to show the level of risk of investments in G10Y DLS and UGCMS DLS by calculating their VaR and TVaR in Section 4. Note that risk measures are calculated under the real-world probability measure, not under the risk-neutral measure. The interest rate models are originally designed for option pricing, so they are defined under the risk-neutral measure. However, as the risk management is gaining more importance, models under the real-world measure are getting more attentions. Section 4 deals with simulations of paths under both of the risk-neutral measure and the real-world measure.

\section{Numerical results}

In financial markets, the no-arbitrage price of a traded instrument is determined by the expectation of the discounted payoff of the instrument under the risk-neutral measure. Thus, the price of a DLS is computed as the expectation of the discounted payoff of the DLS. When we say that the no-arbitrage price of a certain DLS product is 1.05 , for instance, we mean that there would be an arbitrage opportunity if we invest more than or less than 1.05 to get the given payoff of the DLS. We will assume Hull-White model given in equation (3.1) and compute the prices of DLS products of interest by Monte Carlo simulations in this section. We discount the payoff scheduled at time $T$ by multiplying $e^{-R(0, T) T}$ to the payoff where $R(0, T)$ is the spot interest rate prevailing at time 0 for maturity $T$.

\subsection{Calibration and path generation}

In order for numerical analyses of DLS products, we need to determine the parameters of the underlying model. With Hull-White model in equation (3.1), values of $\alpha$ and $\sigma$ have to be determined. These values are parameters under the risk-neutral measure. Many times, calibrating parameter values of the underlying financial model uses the least-squares method. In other words, we find parameter values that minimize the sum of squared differences between the derivative prices observed in the market and ones computed by the model. Since most arbitrage-free approaches for interest rate models fit the parameters to the zero-coupon bond yield curve (Ang and Sherris, 1997), we use the zero-coupon bond prices to calibrate the parameter values under the risk-neutral measure. More specifically, we find the parameters that minimize the following objective function

$$
\sum_{i}\left(U_{i}-V_{i}\right)^{2},
$$

where $U_{i}$ is the $i^{\text {th }}$ bond price observed in the market and $V_{i}$ is the $i^{\text {th }}$ bond price computed from the model. In case of G10Y DLS, German bond prices on May 26, 2019 were used with maturities ranging from zero to 10 years. The market prices were available with maturities 3,6,9 months and from 1 to 10 years by year. In case of UGCMS DLS, we used USD bond prices on September 25, 2018 with maturities 1, 3, 6 months and from 1 to 5 years and GBP bond prices on the same date with maturities 1, 3, 6 months and from 1 to 12 years.

The volatility term $\sigma$ is the same under the risk-neutral measure and the real-world measure as we can see from the well-known Girsanov's theorem. But the drift term which is the coefficient of $d t$ term in equation (3.1) will be different under two different probability measures. To determine the drift term under the real-world measure, the market price of risk should be estimated. It is often assumed to be 0 because it is not clear how to estimate it and the numerical differences between simulations under two 
different probability measures are not really known. However, assuming the market price of risk to be 0 may distort the distribution of simulated paths except cases with very short term horizons. Thus, it would be desirable to estimate the market price of risk in some way. For more details of simulations under the real-world measure, see Hull et al. (2014) or Yasuoka (2015) among others.

In this work, we followed the procedure given in Hull et al. (2014) for simulating paths under the real-world measure as follows. Assume that the underlying model under the risk-neutral measure is given as in equation (3.1). Assuming the constant market price of risk, $\lambda$, the underlying model under the real-world measure can be written as

$$
d r_{t}=\left[\theta(t)-\alpha r_{t}+\lambda \sigma\right] d t+\sigma d z
$$

and $\lambda$ is estimated by

$$
\lambda=-\frac{F(T)-r_{0}}{\sigma T}
$$

where $F(T)$ is the long-run average instantaneous forward rate with maturity $T$ and $r_{0}$ is the continuously compounded long-run average short-term interest rate.

In the risk-neutral world, investors are risk-neutral, but in the real-world, they are not. When the investors are risk-averse in the real world as they usually are, they would require higher return from risky assets, so the drift of the bond price process would be higher under the real-world measure than under the risk-neutral measure. Since the interest rates moves the opposite way from bond prices, the drift of the interest rate model will be smaller under the real-world measure. It implies a negative value of $\lambda$.

There are, in general, two methods for generating paths from Hull-White model, Monte Carlo (MC) method and Hull-White Tree (HWT) method (Hull and White, 1996). MC method has an advantage that it can easily incorporate complicated payoff structures including path-dependent features. On the other hand, HWT method has an advantage in reflecting the mean reversion of interest rates since it has higher degrees of freedom by using trinomial trees. DLS products are typically path-dependent with relatively complex payoff structure, and they can be easily implemented by MC method. Thus, we used MC method and the following is the steps that we followed for the numerical studies.

(1) Calibrate the parameter values under the risk-neutral measure using the least-squares method.

(2) Generate random paths from Hull-White model with calibrated parameter values in (1).

(3) Calculate the payoff for each path and discount it by multiplying $\exp (-R(0, \tau) \tau)$ where $\tau$ is the time of payments.

(4) Take the average of the discounted payoff and use it to approximate the price of DLS.

(5) Compute the market price of risk, $\lambda$ using observed forward rates.

(6) Calculate the drift under the real-world measure using $\lambda$ in (5).

(7) Generate random paths from Hull-White model with parameter values under the real-world measure and compute risk measures.

Figure 7 shows the generated MC paths under the real-world measure: (a) German $10 \mathrm{Y}$ bond rate, (b) USD 5Y CMS rate, and (c) GBP 7Y CMS rate. The number of paths is 10,000 in all three cases. 


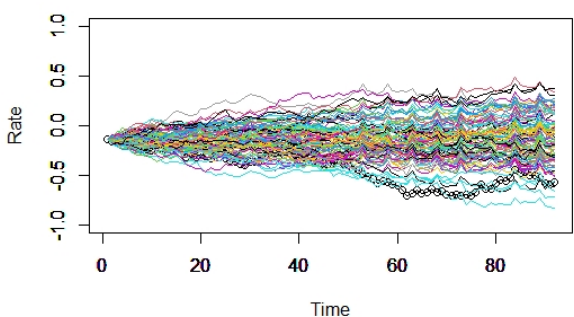

(a) Paths of German $10 Y$ bond rate by MC simulations

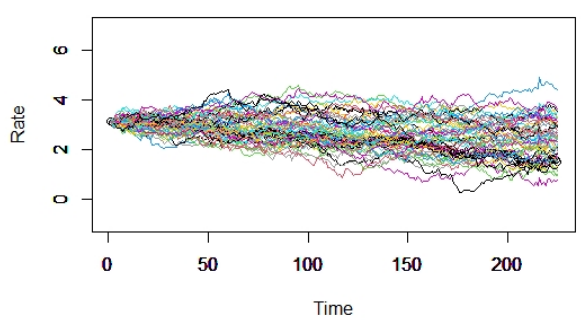

(b) Paths of USD 5Y CMS rate by MC simulations

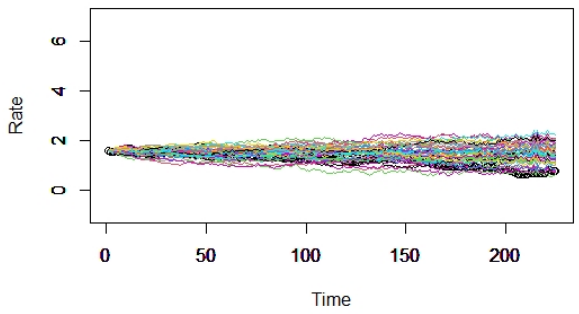

(c) Paths of GBP 7Y CMS rates by MC simulations

Figure 7: Paths of German bond rates and USD-GBP CMS rates by $M C$ simulations.

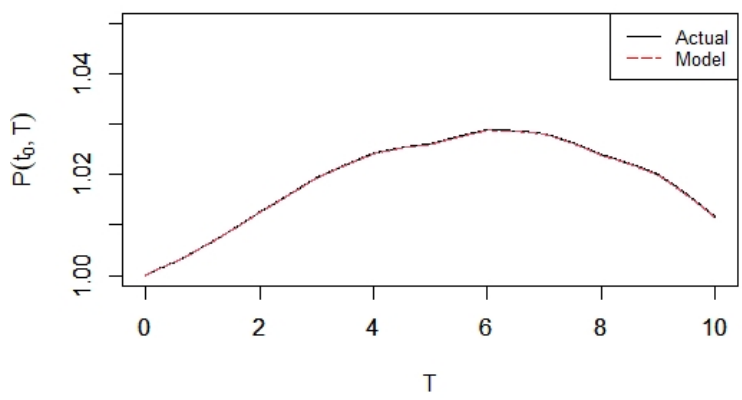

Figure 8: The observed German bond prices and the corresponding model prices; the solid line is the observed prices and dotted line is the model prices.

In each plot, the line with bold dots is the actually observed path. In all three plots, it can be seen that the actually observed path is within the range of the simulated paths, but it is a rather extreme case that is not commonly observed. 
Table 3: The price and the fair coupon rate of G10Y DLS

\begin{tabular}{cc}
\hline \hline Price & Fair coupon rate \\
$0.9662 \times P v$ & $4.76 \%$ \\
\hline \hline
\end{tabular}

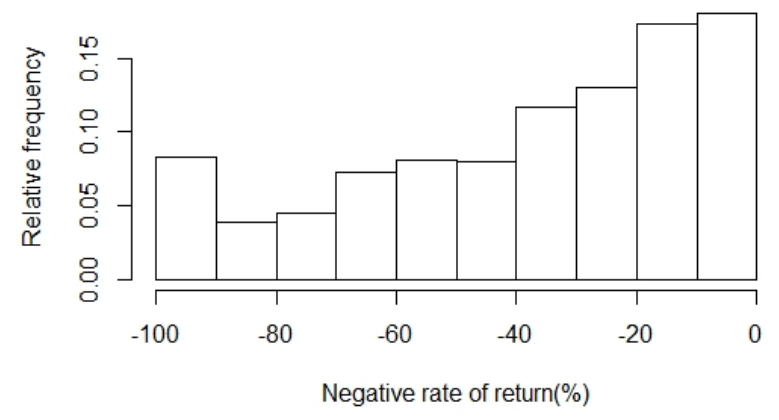

Figure 9: Histogram of relative frequencies of paths with a negative rate of return, G10Y DLS.

Table 4: Counts and relative frequencies of paths with a negative rate of return, G10Y DLS

\begin{tabular}{crc}
\hline \hline Rate of return $(\%)$ & Counts & Relative frequency \\
\hline$-90 \sim-100$ & 178 & $8.28 \%$ \\
$-80 \sim-90$ & 83 & $3.86 \%$ \\
$-70 \sim-80$ & 96 & $4.47 \%$ \\
$-60 \sim-70$ & 157 & $7.31 \%$ \\
$-50 \sim-60$ & 173 & $8.05 \%$ \\
$-40 \sim-50$ & 171 & $7.96 \%$ \\
$-30 \sim-40$ & 251 & $11.68 \%$ \\
$-20 \sim-30$ & 279 & $12.98 \%$ \\
$-10 \sim-20$ & 373 & $17.37 \%$ \\
$0 \sim-10$ & 388 & $18.05 \%$ \\
\hline Total & 2149 & $100 \%$ \\
\hline \hline
\end{tabular}

Table 5: VaR and TVaR of the loss of G10Y DLS

\begin{tabular}{ccccc}
\hline \hline Conf. level & $\mathbf{9 9 . 2 5 \%}$ & $\mathbf{9 9 \%}$ & $\mathbf{9 5 \%}$ & $\mathbf{9 0 \%}$ \\
\hline VaR & $92.96 \%$ & $86.41 \%$ & $37.30 \%$ & $12.27 \%$ \\
TVaR & $97.90 \%$ & $95.76 \%$ & $64.45 \%$ & $44.07 \%$ \\
\hline \hline
\end{tabular}

\subsection{One-asset derivative-linked securities (DLS)}

As mentioned in Section 4.1, we calibrate parameters of Hull-White model using German bond prices on May 25, 2019 with maturities ranging from zero to 10 years. Calibration results from equation (4.1) are $\alpha=0.19, \sigma=0.0036$, and $\lambda=-0.5225$. For pricing, we generate paths from equation (3.1) with $\alpha=0.19$ and $\sigma=0.0036$ and for risk measures, generate paths from equation (4.2) with $\alpha=0.19, \sigma=0.0036$, and $\lambda=-0.5225$. Figure 8 compares the actual bond price and the price computed from the model with calibrated parameters. 
Table 6: Calibration results for UGCMS DLS

\begin{tabular}{|c|c|c|c|c|c|}
\hline \multicolumn{3}{|c|}{ USD 5Y CMS } & \multicolumn{3}{|c|}{ GBP 7Y CMS } \\
\hline$\alpha$ & $\sigma$ & $\lambda$ & $\alpha$ & $\sigma$ & $\lambda$ \\
\hline 0.1898 & 0.0118 & -0.3043 & 0.2655 & 0.0042 & -0.2130 \\
\hline
\end{tabular}

Table 7: The price and the fair coupon rate of UGCMS DLS

\begin{tabular}{cc}
\hline \hline Price & Fair coupon rate \\
$0.9355 \times P v$ & $7.0182 \%$ \\
\hline \hline
\end{tabular}

Table 3 provides the price and fair coupon rate of G10Y DLS calculated by random paths generated under the risk-neutral measure. By the fair coupon rate, we mean the coupon rate that makes the discounted expected payoff equal to the initial investment. If this value is larger than the actual rate, then the product is regarded as favorable to sellers and otherwise, the product is favorable to investors. We computed the fair coupon rate by Newton Raphson method. The price of G10Y DLS is $0.9662 \times P v$ where $P v$ is the initial investment. In other words, when 1 is invested at $t=0$, then its discounted payoff is expected to be 0.9662 . The fair coupon rate was computed as $4.76 \%$, which is considerably higher than the coupon rate actually proposed with G10Y DLS, $1.4 \%$.

Next, we investigate the distribution of sample paths under the real-world measure. Figure 9 is a histogram of the relative frequencies of paths with negative rate of returns and Table 4 contains counts of paths with negative rate of returns. The distribution of paths with negative rate of returns is skewed to the left with a small spike at the leftmost interval. The spike is due to the lower bound of the loss; the maximum possible loss is $98.6 \%$ of the initial investment. Among 10,000 sample paths generated under the risk-neutral measure, 2149 paths ended up with payoffs less than the original investment and 135 paths with the minimum possible payoffs, respectively. The actual path realized in year 2018 tends to be an extreme case; however, the probability of observing paths with the payoff less than the original investment is $21.49 \%$, which is not small.

Table 5 contains values of VaR and Tail-VaR of the loss. For example, $90 \%$ VaR is $12.27 \%$, which means that the probability of observing more serious loss than $12.27 \%$ of the initial investment is 10\%. $90 \%$ TVaR, the expected loss given that loss is greater than $90 \% \mathrm{VaR}$ is $44.07 \%$ of the initial investment. With the confidence level of $99.25 \%$, VaR is $92.96 \%$ and TVaR is $97.9 \%$. Thus, this DSL product does not seem to be a low-risk instrument at all.

\subsection{Two-asset derivative-linked securities (DLS)}

We calibrate parameters of Hull-White model using USD bond prices and GBP bond prices as mentioned in Section 4.1. USD bond prices used are the ones observed on September 24, 2018 with maturities from zero to five years, and GBP bond prices used are the ones observed on September 25, 2018 with maturities from zero to twelve years. Table 6 shows the calibration results for USD 5Y CMS and GBP 7Y CMS. Figure 10 compares the observed bond prices and the prices computed from the model with the calibrated parameters for USD bonds and GBP bonds. We can see that the observed bond prices and the model prices are very close.

Table 7 shows the price and fair coupon rate of UGCMS DLS calculated by random paths generated under the risk-neutral measure. The price is computed as $0.9355 \times P v$ where $P v$ is the initial investment, and the fair coupon rate is $7.0182 \%$, which is fairly larger than the actual coupon rate, $3.5 \%$. If $7.0182 \%$ is used instead of $3.5 \%$ in the UGCMS DLS, then the no-arbitrage price would be $100 \%$ of the initial investment.

Next, we analyze the distribution of paths under the real-world probability measure. Figure 11 


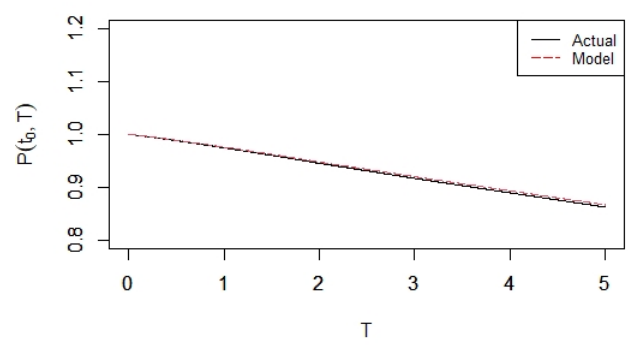

(a) USD bond price

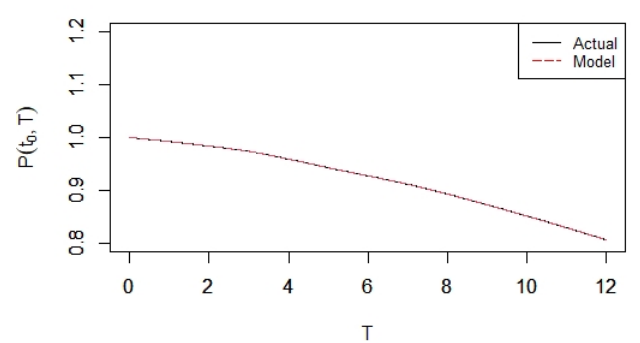

(b) GBP bond price

Figure 10: Plot comparing the actual and theoretical bond price.

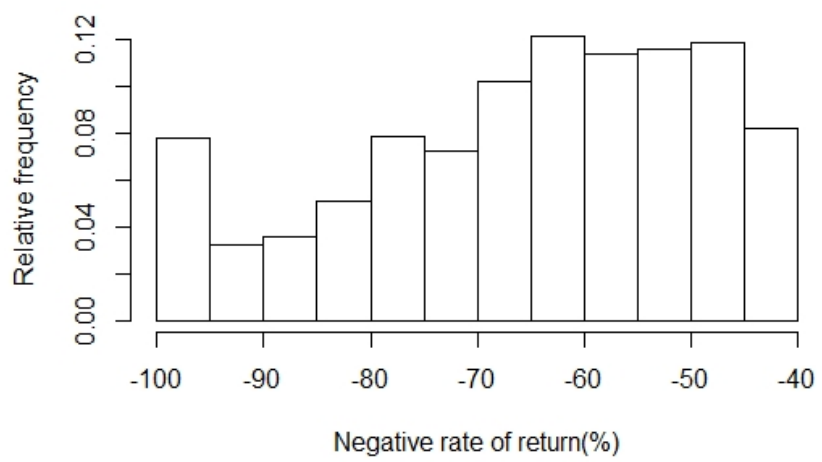

Figure 11: Histogram of relative frequencies of paths with a negative rate of return, UGCMS DLS.

is the histogram of relative frequencies of paths with negative returns and Table 8 shows the counts and relative frequencies of paths with negative returns. Note that the negative rate of return starts from $-41.5 \%$ and the worst rate of return is $-96.5 \%$ from Figure 5. 1742 and 1538 paths out of 10,000 generated paths ended up with payoffs less than the original investment and less than 53.54\% of the original investment, respectively. Payoff of $53.54 \%$ of the original investment means the rate of return is $-46.46 \%$, which is the actual realized rate of return of UGCMS DLS. The probability of obtaining the payoff with the rate of return less than $-46.46 \%$ is $15.38 \%$, so the realized payoff can be considered to be in the range of probable cases.

Table 9 provides the values of VaR and TVaR of the loss of UGCMS DLS. For example, $90 \%$ VaR and TVaR are $58.67 \%$ and $76.75 \%$, respectively. Thus, the probability of observing a loss higher than $58.67 \%$ of the initial investment is $10 \%$ and the expected loss given that loss is greater than $90 \%$ $\mathrm{VaR}$ is $76.75 \%$ of the initial investment. With the confidence level of $98.75 \%$, VaR is $95.84 \%$ and TVaR is $96.48 \%$. As with the case of G10Y DLS, UGCMS DLS also does not seem to be a low-risk instrument. 
Table 8: Counts and relative frequencies of paths with a negative rate of return, UGCMS DLS

\begin{tabular}{ccc}
\hline \hline Rate of return $(\boldsymbol{\%})$ & Counts & Relative frequency \\
\hline$-90 \sim-100$ & 191 & $10.96 \%$ \\
$-80 \sim-90$ & 150 & $8.61 \%$ \\
$-70 \sim-80$ & 263 & $15.10 \%$ \\
$-60 \sim-70$ & 389 & $22.33 \%$ \\
$-50 \sim-60$ & 400 & $22.96 \%$ \\
$-40 \sim-50$ & 349 & $20.03 \%$ \\
Total & 1742 & $100.00 \%$ \\
\hline \hline
\end{tabular}

Table 9: VaR and TVaR of the loss of UGCMS DLS

\begin{tabular}{ccccc}
\hline \hline Conf. level & $\mathbf{9 8 . 7 5 \%}$ & $\mathbf{9 7 \%}$ & $\mathbf{9 5 \%}$ & $\mathbf{9 0 \%}$ \\
\hline VaR & $95.84 \%$ & $84.81 \%$ & $73.98 \%$ & $58.67 \%$ \\
TVaR & $96.48 \%$ & $93.05 \%$ & $87.43 \%$ & $76.75 \%$ \\
\hline \hline
\end{tabular}

\section{Conclusion}

Recently, the proportion of contracts and trading volumes of DLS in financial markets in South Korea is increasing. For five years from year 2014, the proportion of DLS contracts to ELS contracts increased from $12 \%$ to $20 \%$, and the proportion of trading volumes increased from $16 \%$ to $23 \%$. Meanwhile, in 2018 and 2019, some DLS products in the Korean market have caused huge losses to investors. In this regard, we evaluated prices and fair coupon rates as well as VaR and TVaR of DLS products: G10Y DLS and UGCMS DLS, which were at the center of social issues in Korea a few years ago. Although we investigated two DLS products only numerically, this study would be meaningful in the respect that there are not much research on interest rate-linked DLS.

We used Hull-White model for the underlying interest rate model. Hull-White model is, as a noarbitrage model, known to have an advantage of being able to accurately fit the initial term structure. For pricing and finding fair coupon rates, we calibrated parameters of Hull-White model under the risk-neutral measure but for risk measures, calibrated them under the real-world probability measure. After calibrating parameters, we generated paths through Monte Carlo methods.

With both of G10Y DLS and UGCMS DLS, the no-arbitrage prices were computed to be lower than the initial investments, and the fair coupon rates were computed to be higher than the actual coupon rates. In other words, the coupon rates offered by financial institutions were unfavorable to investors, assuming that the undelrying asset followed Hull-White model.

The actual realized losses of G10Y DLS and UGCMS DLS were $98.6 \%$ and $46.46 \%$ of original investments, respectively. From the distribution of paths generated under the real-world measure, we could see that the realized path of UGCMS DLS was in the range of probable cases, while that of G10Y DLS was rather an extreme case. From the estimated risk measures, both of DLS products did not seem to be low-risk instruments.

Short rate models are mostly developed for pricing and analyzed under the risk-neutral measure. Thus, the risk-neutral simulation under Hull-White model is widely performed, but the simulation under the real-world probaiblity measure is not common and quite challenging. We followed a method of finding parameter values under the real-world measure given in Hull et al. (2014), but this method is, in fact, not very rigorous. It would be another possibility to try other models such as the LIBOR market model (Brace et al, 1997) that models forward rates that can be directly observd in financial markets. We would like to leave this as a future research topic. 


\section{References}

Ang A and Sherris M (1997). Interest rate risk management: Developments in interest rate term structure modeling for risk management and valuation of interest-rate-dependent cash flows', North American Actuarial Journal, 1, 1-26.

Brace A, Gatarek D, and Musiela M (1997). The Market Model of Interest Rate Dynamics, Mathematical Finance, 7, 127-154.

Broadie M, Glasserman P, and Kou S (1997). A continuity correction for discrete barrier options, Mathematical Finance, 7, 325-349.

Cox JC, Ingersoll JE, and Ross SA (1985). A theory of the term structure of interest rates, Econometrica, $53,385-407$.

Gupta A and Subrahmanyam MG (2005). Pricing and hedging interest rate options: evidence from cap-floor markets, Journal of Banking and Finance, 29, 701-733.

Henrard M (2009). Efficient Swaptions Price in Hull-White One Factor Model, arXiv 0901.1776v1.

Ho TSY and Lee SB (1986). Term structure movements and pricing interest rate contingent claims, Journal of Finance, 41, 1011-1029.

Hull J and White A (1990). Pricing interest rate derivative securities, Review of Financial Studies, 3, $573-592$.

Hull J and White A (1996). Using Hull-White interest rate trees, Journal of Derivatives, 3, 26-36.

Hull J (2011). Options, Futures, and Other Derivatives (8th ed), Pearson, London.

Hull J, Sokol A, and White A (2014). Short rate joint measure models, Risk, October 2014, 59-63.

Jeong D, Wee IS, and Kim JS (2010). An operator splitting method for pricing the ELS option, Journal of the Korean Society for Industrial and Applied Mathematics, 14, 175-187.

Klugman SA, Panjer HH, and Willmot GE (2012). Loss Models: From Data to Decisions (4th ed), John Wiley \& Sons, New York.

Lee H, Ko B, and Song S (2019). Valuing step barrier options and their icicled variations, North American Journal of Economics and Finance, 49, 396-411.

Lyuu YD (1998). Very fast algorithms for barrier option pricing and the ballot problem, Journal of Derivatives, 5, 68-79.

Ostrovski V (2013). Efficient and exact simulation of the Hull-White model, SSRN Electronic Journal, 10.2139/ssrn.2304848.

Rendleman RJ and Bartter BJ (1980). The pricing of options on debt securities, Journal of Financial and Quantitative Analysis, 15, 11-24.

Vasicek OA (1977). An equilibrium characterization of the term structure, Journal of Financial Economics, 5, 177-188.

Yasuoka T (2015). Interest-rate simulation under the real-world measure within a Gaussian HJM framework, Quantitative Finance Letters, 3, 10-16. 
